\title{
Translation averages of dyadic weights are not always good weights
}

Lesley A. Ward

\begin{abstract}
The process of translation averaging is known to improve dyadic BMO to the space BMO of functions of bounded mean oscillation, in the sense that the translation average of a family of dyadic BMO functions is necessarily a BMO function. The present work investigates the effect of translation averaging in other dyadic settings. We show that translation averages of dyadic doubling measures need not be doubling measures, translation averages of dyadic Muckenhoupt weights need not be Muckenhoupt weights, and translation averages of dyadic reverse Hölder weights need not be reverse Hölder weights. All three results are proved using the same construction.
\end{abstract}

\section{Introduction.}

Several important function spaces on the real line, such as the space $\mathrm{BMO}$ of functions of bounded mean oscillation, Muckenhoupt's spaces of $A_{p}$ weights, and the spaces of reverse Hölder weights, are defined in terms of properties that must hold on every real interval. Such function spaces have less restrictive dyadic versions, in which the defining property is only

2000 Mathematics Subject Classification: 42B35, 42B25.

Keywords: Doubling measures, dyadic weights, $A_{p}$ weights, reverse Hölder weights, Muckenhoupt weights, translation average. 
required to hold on dyadic intervals. These dyadic versions then contain the original function spaces. Similarly, the space of doubling measures, defined by requiring the ratio $\mu(I) / \mu(J)$ to be uniformly bounded for all pairs of adjacent intervals $I$ and $J$ of equal length, has a dyadic version, in which the doubling property need only hold for dyadic sister intervals.

Averaging a collection of functions tends to smooth out irregularities, producing a better-behaved function. In this paper we consider the following averaging process. The translation average $\varphi$ of a collection of functions $\left\{\varphi_{t}\right\}$ on the unit interval with endpoints identified, indexed by $t \in[0,1]$, is defined by

$$
\varphi(x)=\int_{0}^{1} \varphi_{t}(x+t) d x
$$

The translation average of a collection of measures is defined analogously.

Garnett and Jones showed in [GJ] that the translation average of a collection of dyadic BMO functions is necessarily a BMO function. (This is not true for the usual average.) Specifically, if $t \longrightarrow \varphi_{t}$ is a measurable mapping from $\mathbb{R}^{m}$ to the space of dyadic BMO functions such that all $\varphi_{t}$ are supported on a fixed dyadic cube, the dyadic BMO constant of each $\varphi_{t}$ is bounded by 1 , and each $\varphi_{t}$ has mean zero, then the function

$$
\varphi^{N}(x)=\frac{1}{(2 N)^{m}} \int_{\left|t_{j}\right| \leq N} \varphi_{t}(x+t) d t
$$

is in BMO for each $N$, with uniform BMO constant. They exploit this idea to give new proofs of theorems on the structure of BMO functions and on the distance to $L^{\infty}$ in BMO, and of the factorization theorem for $A_{p}$ weights, by first obtaining each theorem in the easier dyadic case, and then averaging the results of the dyadic decomposition over translations. In this paper we investigate whether the translation averaging process has the same "improving" effect in other settings.

We prove that translation averaging does not always improve dyadic spaces. First, there are families of dyadic doubling measures, with uniform and arbitrarily small dyadic doubling constant, whose translation averages are not doubling measures.

Theorem 1.1. Given $C>1$, there is a family of dyadic doubling measures $\left\{\mu_{t}\right\}_{t \in[0,1]}$ on $[0,1]$, with dyadic doubling constant at most $C$ for all $t \in$ 
$[0,1]$, such that the translation average

$$
\mu(\cdot)=\int_{0}^{1} \mu_{t}(\cdot+t) d t
$$

is not a doubling measure.

Second, for each of Muckenhoupt's $A_{p}$ spaces, there are families of dyadic $A_{p}$ weights on the unit interval, with uniform and arbitrarily small dyadic $A_{p}$ constant, whose translation averages are not $A_{p}$ weights.

Theorem 1.2. For each real $p$ with $1 \leq p \leq \infty$ and for each $C>1$, there is a family of $A_{p}^{d}$ weights $\left\{w_{t}\right\}_{t \in[0,1]}$ on $[0,1]$, with $\left\|w_{t}\right\|_{A_{p}^{d}} \leq C$ for all $t \in[0,1]$, such that the translation average

$$
w(x)=\int_{0}^{1} w_{t}(x+t) d t
$$

is not an $A_{p}$ weight.

Third, for each reverse Hölder space $R H_{q}$, there are families of dyadic reverse Hölder- $q$ weights on the unit interval, with uniform and arbitrarily small dyadic reverse Hölder- $q$ constant, whose translation averages are not reverse Hölder weights.

Theorem 1.3. For each real $q$ with $1<q<\infty$ and for each $C>1$, there is a family of $R H_{q}^{d}$ weights $\left\{w_{t}\right\}_{t \in[0,1]}$ on $[0,1]$, with $\left\|w_{t}\right\|_{R H_{q}^{d}} \leq C$ for all $t \in[0,1]$, such that the translation average

$$
w(x)=\int_{0}^{1} w_{t}(x+t) d t
$$

is not a $R H_{q}$ weight.

Doubling measures, Muckenhoupt's classes of $A_{p}$ weights, reverse Hölder weights, and their dyadic versions are defined in Section 2.

All three results are proved by the same construction, which is developed in Theorem 1.4 below. We construct a family of weights $\left\{w_{t}\right\}$, $0 \leq t \leq 1$, depending on a parameter $\alpha, 0<\alpha<1$, with the following properties. The weights are the densities of dyadic doubling measures $\mu_{t}$. The weights lie in every $A_{p}^{d}$ space, and in every $R H_{q}^{d}$ space for which 
$q<1 / \alpha$, and so by decreasing the parameter $\alpha$ we can ensure that the weights lie in any given $R H_{q}^{d}$ space. Moreover, by decreasing $\alpha$, we can also make the dyadic doubling constant, the $A_{1}^{d}$ constant, the $A_{p}^{d}$ constant, and the $R H_{q}^{d}$ constant arbitrarily close to 1 . However, the measure $\mu$ whose density is the translation average $w(x)=\int_{0}^{1} w_{t}(x+t) d t$ of the weights is not a doubling measure, which proves Theorem 1.1.

On the other hand, measures whose densities are $A_{p}$ weights or reverse Hölder weights are necessarily doubling; this follows from the reverse Hölder property and $\cup_{p \geq 1} A_{p}=\cup_{q>1} R H_{q}$. Therefore $w(x)$ is not in $A_{p}$ for any $p$, nor in $R H_{q}$ for any $q$, establishing Theorems 1.2 and 1.3.

In order to state the main theorem precisely, we first make some definitions.

Let $\alpha$ be a real number with $0<\alpha<1$. Let $\beta$ be a rational number such that

$$
0<\beta<\frac{\alpha}{\alpha+1} \quad \text { and } \quad \beta+\alpha<1 .
$$

The first condition implies that $\alpha-\beta \alpha-\beta>0$. Let $\left\{N_{j}\right\}_{j=1}^{\infty}$ be a sequence of rapidly increasing integers, chosen so that $N_{j} \beta$ is an integer for all $j$, so that $N_{1} \geq 1 / \beta$ and $N_{2} \geq 2 / \beta$, and so that

$$
N_{j} \geq \frac{1}{\alpha-\beta \alpha-\beta}\left(N_{1}+\cdots+N_{j-1}\right), \quad \text { for } j=2,3, \ldots
$$

For example, when $\alpha=1 / 2$ and $\beta=1 / 4$, the sequence $N_{1}=4, N_{j}=$ $8(9)^{j-2}$ for $j \geq 2$ satisfies these conditions. In general, a sequence satisfying $N_{j} \geq C\left(N_{1}+\cdots+N_{j-1}\right)$ must grow exponentially, since $N_{j} \geq$ $C(1+C)^{j-1} N_{1}$.

We identify the unit circle with the interval $[0,1]$. The dyadic intervals in $[0,1]$ comprise the set

(8) $\mathcal{D}=\left\{I=\left[\frac{j}{2^{k}}, \frac{j+1}{2^{k}}\right): j, k\right.$ integers, $\left.0 \leq j \leq 2^{k}-1, k=0,1,2, \ldots\right\}$

of half-open dyadic subintervals of $[0,1]$. Let $\mathcal{D}_{N}$ denote the collection of intervals in $\mathcal{D}$ of length $2^{-N}$, for $N=0,1,2, \ldots$ We use the term dyadic sisters for the two halves or daughters of a single dyadic interval, their parent. Any two dyadic intervals are either nested or disjoint.

Define the generational distance $d(I, J)$ between two dyadic intervals $I$ and $J$ of equal length by

$$
d(I, J)=\log _{2}\left(\frac{|K|}{|I|}\right)
$$


where $K$ is the smallest dyadic interval containing both $I$ and $J$. Thus $d(I, I)=0$, and in general $d(I, J)$ is the number of generations back to the first dyadic common ancestor of $I$ and $J$.

Given a point $t \in[0,1]$, and $j \geq 1$, let $I_{t}^{j}$ be the unique dyadic interval in $\mathcal{D}_{N_{j}}$ which contains $t$. Label the dyadic intervals in $\mathcal{D}_{N_{j}}$ immediately to the right of $I_{t}^{j}$ as $J_{t}^{j}, K_{t}^{j}, L_{t}^{j}$, and $M_{t}^{j}$, reading from left to right. When $t$ is very near the right end of $[0,1]$, some or all of $J_{t}^{j}, K_{t}^{j}, L_{t}^{j}$, and $M_{t}^{j}$ are wrapped around to the left end of $[0,1]$.

On the unit circle, $K_{t}^{j}$ is the translation of $I_{t}^{j}$ to the right by $2\left|I_{t}^{j}\right|$. We define a nested, decreasing sequence $\left\{\mathcal{G}_{j}\right\}_{j=1}^{\infty}$ of sets in $[0,1]$ by letting $\mathcal{G}_{0}=[0,1]$, and for $j \geq 1$ letting

$$
\begin{aligned}
\mathcal{G}_{j} & =\left\{t \in \mathcal{G}_{j-1}: d\left(I_{t}^{j}, K_{t}^{j}\right)=N_{j} \beta\right\} \\
& =\left\{t \in[0,1]: d\left(I_{t}^{1}, K_{t}^{1}\right)=N_{1} \beta, \ldots, d\left(I_{t}^{j}, K_{t}^{j}\right)=N_{j} \beta\right\} .
\end{aligned}
$$

For instance, $t \in \mathcal{G}_{2}$ if the generational distance from the dyadic interval $I_{t}^{1}$ of length $2^{-N_{1}}$ containing $t$, to the dyadic interval $K_{t}^{1}$ which is the translation of $I_{t}^{1}$ to the right by twice the length of $I_{t}^{1}$, is exactly $N_{1} \beta$, and if in addition $d\left(I_{t}^{2}, K_{t}^{2}\right)$ is exactly $N_{2} \beta$.

Define a family $\left\{w_{t}\right\}_{t \in[0,1]}$ of weights on the unit circle as follows. For $t \in \mathcal{G}_{0} \backslash \mathcal{G}_{1}$, let $w_{t} \equiv 1$. For $t \in \mathcal{G}_{j} \backslash \mathcal{G}_{j+1}, j \geq 1$, let

$$
w_{t}(x)= \begin{cases}\left(M_{d}\left(c \delta_{t}(x)\right)\right)^{\alpha}, & x \notin I_{t}^{j}, \\ 2^{N_{j} \alpha}, & x \in I_{t}^{j} .\end{cases}
$$

Here $c=\left(2-2^{\alpha}\right)^{1 / \alpha}, \delta_{t}$ is the Dirac delta function centred at $t, I_{t}^{j}$ is the unique dyadic interval of length $2^{-N_{j}}$ that contains $t$, and $M_{d}$ is the dyadic maximal operator, defined by

$$
M_{d}(f)(x)=\sup _{\substack{I \ni x \\ I \in \mathcal{D}}} \frac{1}{|I|} \int_{I}|f(y)| d y .
$$

The weight $w_{t}$ is constant on each dyadic interval of length $2^{N_{j}}$, and decays away from its maximum, taken on the dyadic interval $I_{t}^{j}$ containing $t$, as $|x-t|$ increases.

For each $j$, let $A^{j}$ be the interval of length $2^{-N_{j}}$ centred at 0 , and let $B^{j}$ be the interval of the same length obtained by translating $A^{j}$ to the right by the distance $3\left|A^{j}\right|$. These intervals $A^{j}$ and $B^{j}$ are not dyadic. 
Theorem 1.4. Define the numbers $\alpha, \beta$, and $\left\{N_{j}\right\}_{j=1}^{\infty}$, the sets $\left\{\mathcal{G}_{j}\right\}_{j=1}^{\infty}$, the weights $\left\{w_{t}\right\}_{t \in[0,1]}$, and the intervals $\left\{A^{j}\right\}_{j=1}^{\infty}$ and $\left\{B^{j}\right\}_{j=1}^{\infty}$ as above. Then the weights $w_{t}$ are dyadic $A_{p}$ weights, with uniform $A_{p}^{d}$ constant, for $1 \leq p \leq \infty$, and they are dyadic reverse Hölder weights, with uniform $R H_{q}^{d}$ constant, for $1<q<1 / \alpha$. Also, the associated measures $\mu_{t}$ with densities $w_{t}$ are dyadic doubling measures with uniform dyadic doubling constant. All these constants converge to 1 as $\alpha$ decreases to 0 . Finally, the translation average $\mu(\cdot)=\int_{0}^{1} \mu_{t}(\cdot+t) d t$ of the measures $\mu_{t}$ satisfies

$$
\frac{\mu\left(A^{j}\right)}{\mu\left(B^{j}\right)} \geq C 2^{N_{j} \beta \alpha} \longrightarrow \infty, \quad \text { as } j \longrightarrow \infty,
$$

and therefore $\mu$ is not a doubling measure.

Remark. We mention that it is possible to prove Theorem 1.1 using a completely different family of examples of dyadic doubling measures on the unit interval. One fixes $r, s$ such that $0<s<r<1$ and $r+s=1$, and defines the measures $\mu_{t}$ recursively, first assigning mass $r$ to either the left or right half of $[0,1]$ and mass $s$ to the other half. Next, one assigns the fraction $r$ of the mass of $[0,1 / 2)$ to either the left or right half of $[0,1 / 2)$ and the fraction $s$ to the other half, and similarly for $[1 / 2,1]$, and so on at smaller and smaller scales, producing dyadic doubling measures with uniform dyadic doubling constant $r / s$. However, the details of making the ensemble of left/right choices so that the resulting translation average $\mu$ is not doubling make the construction quite intricate.

I am grateful to a referee and to David Cruz-Uribe for pointing out related work of Petermichl, Nazarov, Treil, and Volberg. In [P], Petermichl obtains the kernel of the one-dimensional Hilbert transform as the result of an averaging process over kernels of dyadic shift operators. Her averaging process involves both dilations and translations of the standard dyadic grid. She applies the decomposition to show that the commutator of the Hilbert transform with matrix multiplication by a BMO matrix of size $n \times n$ is bounded by a multiple of $\log n$ times the BMO-norm of the matrix, and, with Pott $[\mathrm{PP}]$, to prove an analogue of Burkholder's theorem for operator-weighted spaces. Namely, for an operator weight function $W$ taking values in the bounded linear operators on a Hilbert space $\mathcal{H}$, if the dyadic martingale transforms are uniformly bounded on $L_{\mathbb{R}}^{2}(W)$ for every dilated and translated dyadic grid in $\mathbb{R}$, then the Hilbert transform is bounded on $L_{\mathbb{R}}^{2}(W)$.

Nazarov, Treil, and Volberg [NTV] use dyadic martingale techniques and an averaging process on dyadic lattices to extend the following well 
known result to the case where $\mu$ is not doubling. Let $T$ be an operator of Calderón-Zygmund type associated to a one-dimensional standard kernel $K$ in $\mathbb{R}^{2}$, satisfying the antisymmetry condition $K(x, y)=-K(y, x)$, for all $x, y \in \mathbb{R}^{2}$. Let $\mu$ be a positive Radon measure such that $\mu(Q) \leq C \ell(Q)$ for all squares $Q$, where $\ell(Q)$ is the side-length of $Q$. Then $T: L^{2}(\mu) \longrightarrow L^{2}(\mu)$ is bounded if and only if the $L^{2}$ estimate holds for characteristic functions of squares. They generalize to operators $T$ which are not antisymmetric, and to higher dimensions.

In Section 2 we recall the definitions of doubling measures, Muckenhoupt's $A_{p}$ spaces, the reverse Hölder spaces, and their dyadic versions. In Section 3 we define the weights $v_{t}=\left(M_{d}\left(c \delta_{t}(x)\right)\right)^{\alpha}$ in terms of dyadic maximal functions of Dirac delta functions, and compute the dyadic $A_{p}$ constants and the dyadic reverse Hölder constants of these weights and the dyadic doubling constants for the associated measures. We also compute the analogous constants for the truncated weights $w_{t}$ defined in (11). In Section 4 we prove our main result, Theorem 1.4.

\section{Background.}

A positive measure $\mu$ on the real line is called a dyadic doubling measure if there is a constant $C \geq 1$ such that

$$
\frac{1}{C} \leq \frac{\mu(I)}{\mu(J)} \leq C
$$

for all pairs $I, J$ of dyadic sister intervals. The smallest such constant $C$ is called the dyadic doubling constant of $\mu$. Equivalently, there is a constant $C^{\prime} \geq 1$ such that for every dyadic interval $I, \mu(\widetilde{I}) \leq C^{\prime} \mu(I)$, where the interval $\widetilde{I}$ is the dyadic parent of $I$. When there is a constant $C \geq 1$ such that (14) holds for every pair $I, J$ of adjacent intervals of equal length, not only for dyadic sisters, $\mu$ is called a doubling measure.

We say that a positive locally integrable function $w$ on the real line is a dyadic $A_{p}$ weight, written $w \in A_{p}^{d}$, for real $p$ with $1<p<\infty$, if

$$
\sup _{I \in \mathcal{D}}\left(\frac{1}{|I|} \int_{I} w\right)\left(\frac{1}{|I|} \int_{I}\left(\frac{1}{w}\right)^{1 /(p-1)}\right)^{p-1}<\infty .
$$

We say $w$ is a dyadic $A_{1}$ weight, written $w \in A_{1}^{d}$, if

$$
\sup _{I \in \mathcal{D}}\left(\frac{1}{|I|} \int_{I} w\right)\left(\operatorname{ess}_{I} \sup _{I} \frac{1}{w}\right)<\infty \text {. }
$$


The suprema in equations (15) and (16) are called the dyadic $A_{p}$ constant and the dyadic $A_{1}$ constant, respectively, of the weight $w$, and are denoted by $\|w\|_{A_{p}^{d}}$ and $\|w\|_{A_{1}^{d}}$. When the suprema in equations (15) and (16) are taken over all real intervals, not just over the dyadic intervals, we recover the definitions of Muckenhoupt's original $A_{p}$ and $A_{1}$ weights.

We say $w$ is a dyadic reverse Hölder-q weight, written $w \in R H_{q}^{d}$, for real $q$ with $1<q<\infty$, if the measure $\mu$ whose density is $w$ is a dyadic doubling measure, and if there is a constant $C \geq 1$ such that for all dyadic intervals $I \in \mathcal{D}$,

$$
\left(\frac{1}{|I|} \int_{I} w^{q}\right)^{1 / q} \leq C \frac{1}{|I|} \int_{I} w
$$

The smallest such constant $C$ is called the dyadic reverse Hölder- $q$ constant of the weight $w$, and is denoted by $\|w\|_{R H_{q}^{d}}$. The original reverse Hölder- $q$ spaces $R H_{q}$ are defined by requiring (17) to hold for all real intervals $I$, not only for dyadic intervals. In the non-dyadic setting $R H_{q},(17)$ implies that the associated measure $\mu$ is doubling $[\mathrm{CF}],[\mathrm{GC}-\mathrm{RF}]$. In the dyadic setting $R H_{q}^{d}$, this is not true [B], and we impose the additional condition that $\mu$ is a dyadic doubling measure so that the dyadic and non-dyadic theories will be parallel.

The dyadic doubling constant and the constants $\|w\|_{A_{p}^{d}},\|w\|_{A_{1}^{d}}$, and $\|w\|_{R H_{q}^{d}}$ are necessarily greater than or equal to 1 .

The canonical examples of weights are the functions $w(x)=|x|^{\alpha}$, for which $w \in A_{\infty}^{d}$ if and only if $\alpha>-1, w \in A_{p}^{d}$ if and only if $-1<\alpha<p-1$, $w \in A_{1}^{d}$ if and only if $-1<\alpha \leq 0$, and $w \in R H_{q}^{d}$ if and only if $\alpha>-1 / p$.

Muckenhoupt's spaces of $A_{p}$ weights are well known in connection with two important operators in harmonic analysis. The Hilbert transform and the maximal function are both bounded operators from $L^{p}$ to itself, for $1<p<\infty$. They are bounded from $L^{p}(d \mu)$ to itself if and only if $\mu$ is absolutely continuous with respect to Lebesgue measure and the density of $\mu$ is an $A_{p}$ weight [M], [HMW]. The spaces of $A_{p}$ weights are nested and increasing with $p$, and their union is denoted by $A_{\infty}$. The reverse Hölder spaces are nested and decreasing as $q$ increases, $1<q<\infty$, and their union is also $A_{\infty}$. The reverse Hölder spaces are also known as the $B_{p}$ spaces. In $[\mathrm{FKP}]$ and $[\mathrm{B}]$, the spaces $A_{\infty}, A_{p}, R H_{q}$ and their dyadic versions are characterized by summation conditions. The $A_{p}$ spaces are also closely related to $\mathrm{BMO}$; if $w \in A_{\infty}$ then $\log w$ is in $\mathrm{BMO}$, and if $\log w$ is in BMO then there is a $\beta>0$ such that $w^{\beta} \in A_{\infty}$. See [GC-RF] for more on the theory of weights. 


\section{Weights from dyadic maximal functions of Dirac deltas.}

In this section we define the particular weights $w_{t}$ used in our construction, and compute their dyadic $A_{p}$ constants, their dyadic reverse Hölder constants, and the dyadic doubling constants of the associated measures $\mu_{t}$.

For most values of $t \in[0,1]$, our weights $w_{t}$ are identically 1 . For the remaining values of $t$, our weights are modified versions of powers of dyadic maximal functions of Dirac delta functions, following the characterization of $A_{1}$ weights by Coifman and Rochberg [CR]. The underlying idea is to produce a large drop in the value of $w_{t}$ at a dyadic point as close as possible to $t$, so that $w_{t}$ is large on the interval $A^{j}+t$ and small on $B^{j}+t$.

Fix a number $\alpha$ with $0<\alpha<1$, and define a normalizing constant $c=\left(2-2^{\alpha}\right)^{1 / \alpha}$. Fix $t \in[0,1]$. Let $\delta_{t}$ denote the Dirac delta function at $t$. Define a weight $v_{t}$ by

$$
v_{t}=\left(M_{d}\left(c \delta_{t}(x)\right)\right)^{\alpha}=\left(\sup _{\substack{I \ni x \\ I \in \mathcal{D}}} \frac{1}{|I|} \int_{I} c \delta_{t}(y) d y\right)^{\alpha} .
$$

See Figure 1. We will see below that the weight $v_{t}(x)$ has a peak at $x=t$, decaying away as $|x-t|$ increases, and that the requirement $\alpha<1$ ensures that $v_{t}$ is integrable. The constant $c=\left(2-2^{\alpha}\right)^{1 / \alpha}$ is chosen so that $v_{t}$ has integral 1.

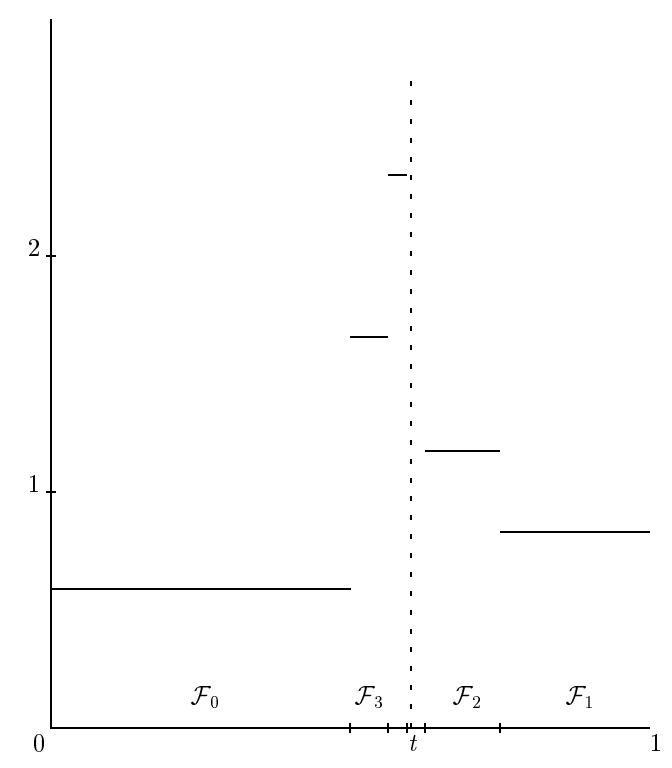

Figure 1. The weight $v_{t}(x)=\left(M_{d}\left(c \delta_{t}(x)\right)\right)^{\alpha}$, with $\alpha=1 / 2$. 
We partition $[0,1]$ into the intervals $\mathcal{F}_{k}$ on which $v_{t}$ is constant. The supremum in (18) is achieved when $I$ is the smallest dyadic interval containing both $x$ and $t$. For $k=0,1,2, \ldots$ let

$$
\begin{aligned}
\mathcal{F}_{k}=\{x \in[0,1]: & |\widehat{I}|=2^{-k}, \text { where } \widehat{I} \text { is the smallest } \\
& \text { dyadic interval containing both } x \text { and } t\} .
\end{aligned}
$$

Then $\left|\mathcal{F}_{k}\right|=2^{-k-1}$. The set $\mathcal{F}_{0}$ is the half of $[0,1]$ that does not contain $t$, $\mathcal{F}_{1}$ is the half of $[0,1] \backslash \mathcal{F}_{0}$ that does not contain $t$, and so on. The weight $v_{t}$ is constant on each $\mathcal{F}_{k}$ : for $x \in \mathcal{F}_{k}$,

$$
v_{t}(x)=\left(M_{d}\left(c \delta_{t}(x)\right)\right)^{\alpha}=\left(\frac{1}{|\widehat{I}|} \int_{\widehat{I}} c \delta_{t}(y) d y\right)^{\alpha}=2^{k \alpha} c^{\alpha} .
$$

Given a weight $v_{t}$, we denote by $\nu_{t}(\cdot)$ the associated measure whose density is $v_{t}$. We use $I_{t}$ to denote dyadic intervals containing the point $t$, and $J$ to denote dyadic intervals not containing $t$. In particular, for the unique dyadic interval $I_{t} \in \mathcal{D}_{N}$ that contains $t$, we have $I_{t}=\cup_{k=N}^{\infty} \mathcal{F}_{k}$.

We collect here some useful properties of the weights $v_{t}$.

Lemma 3.1. Suppose $0<\alpha<1$, and let $c=\left(2-2^{\alpha}\right)^{1 / \alpha}$. Fix $t \in$ $[0,1]$. The weight $v_{t}(x)=\left(M_{d}\left(c \delta_{t}(x)\right)\right)^{\alpha}$ and its associated measure $\nu_{t}(\cdot)=$ $\int . v_{t}(x) d x$ satisfy the following properties.

i) The total mass of $\nu_{t}$ on $[0,1]$ is 1 .

ii) The dyadic interval $I_{t} \in \mathcal{D}_{N}$ containing the point $t$ has mass

$$
\nu_{t}\left(I_{t}\right)=2^{N \alpha-N}
$$

and so the average value of $v_{t}$ on $I_{t}$ is $2^{N \alpha}$.

iii) Any dyadic interval $J \in \mathcal{D}_{N}$ not containing the point $t$ has mass

$$
\nu_{t}(J)=2^{N \alpha-d\left(I_{t}, J\right) \alpha-N} c^{\alpha},
$$

and so the average value of $v_{t}$ on $J$ is $2^{N \alpha-d\left(I_{t}, J\right) \alpha} c^{\alpha}$.

iv) The measure $\nu_{t}$ associated to $v_{t}$ is a dyadic doubling measure, with dyadic doubling constant $2^{\alpha} /\left(2-2^{\alpha}\right)$.

v) The weight $v_{t}$ lies in every dyadic $A_{p}$ space. Its dyadic $A_{1}$ constant is $\left(2-2^{\alpha}\right)^{-1}$. Its dyadic $A_{p}$ constant, for $1<p<\infty$, is

$$
\left(2-2^{\alpha}\right)^{-1}\left(2-2^{\alpha /(p-1)}\right)^{-(p-1)} .
$$


vi) The weight $v_{t}$ lies in all dyadic reverse Hölder-q spaces such that $1<q<1 / \alpha$. Its dyadic reverse Hölder-q constant, for $1<q<1 / \alpha$, is

$$
\left(2-2^{\alpha}\right)\left(2-2^{\alpha q}\right)^{-1 / q}
$$

vii) The dyadic doubling constant, the $A_{1}^{d}$ constant, the $A_{p}^{d}$ constant, and the $R H_{q}^{d}$ constant of $v_{t}$ decrease to 1 as $\alpha$ decreases to 0 .

Before proving Lemma 3.1 we define the truncated version $w_{t}$ of the weight $v_{t}$, and state its properties in Lemma 3.3.

Definition 3.2. Let $L$ be an integer greater than 0 . Let $I_{t}^{L}$ denote the unique dyadic interval of length $2^{-L}$ containing $t$. The truncation of $v_{t}$ at level $L$ is the new weight $w_{t}$ defined by

$$
w_{t}(x)= \begin{cases}\left(M_{d}\left(c \delta_{t}(x)\right)\right)^{\alpha}, & x \notin I_{t}^{L}, \\ 2^{L \alpha}, & x \in I_{t}^{L} .\end{cases}
$$

Denote by $\mu_{t}$ the measure whose density is $w_{t}$; so $\mu_{t}(\cdot)=\int . w_{t}(x) d x$.

The only difference between $v_{t}$ and $w_{t}$ is that $v_{t}$ has been replaced on $I_{t}^{L}$ by its average value on $I_{t}^{L}$. In other words, the "peak" of $v_{t}$ around $t$ has been lopped off, and replaced by the constant value $2^{L \alpha}$ on $I_{t}^{L}$. The truncated weight $w_{t}$ has the same dyadic doubling constant and $A_{1}^{d}$ constant as $v_{t}$, and smaller $A_{p}^{d}$ and $R H_{q}^{d}$ constants than $v_{t}$.

Lemma 3.3. Suppose $0<\alpha<1$, and let $c=\left(2-2^{\alpha}\right)^{1 / \alpha}$. Fix $t \in[0,1]$. Let $w_{t}$ be the weight given by truncating $v_{t}$ at level $L$,

$$
w_{t}(x)= \begin{cases}\left(M_{d}\left(c \delta_{t}(x)\right)\right)^{\alpha}, & x \notin I_{t}^{L}, \\ 2^{L \alpha}, & x \in I_{t}^{L},\end{cases}
$$

where $t \in I_{t}^{L} \in \mathcal{D}_{L}$. Then $w_{t}$ and its associated measure $\mu_{t}(\cdot)=\int w_{t}(x) d x$ satisfy the following properties.

i) The total mass of $\mu_{t}$ on $[0,1]$ is 1 .

ii) The dyadic interval $I_{t} \in \mathcal{D}_{N}$ containing the point $t$ has mass

$$
\mu_{t}\left(I_{t}\right)= \begin{cases}2^{N \alpha-N}, & \text { if } 0 \leq N \leq L\left(\text { so } I_{t} \supseteq I_{t}^{L}\right), \\ 2^{L \alpha-N}, & \text { if } N>L\left(\text { so } I_{t} \subset I_{t}^{L}\right) .\end{cases}
$$


Therefore the average value of $w_{t}$ on $I_{t}$ is $2^{N \alpha}$ if $0 \leq N \leq L$, and $2^{L \alpha}$ if $N>L$.

iii) Any dyadic interval $J \in \mathcal{D}_{N}$ not containing the point $t$ has mass

$$
\mu_{t}(J)= \begin{cases}2^{N \alpha-d\left(I_{t}, J\right) \alpha-N} c^{\alpha}, & \text { if } J \not \subset I_{t}^{L}, \\ 2^{L \alpha-N}, & \text { if } J \subset I_{t}^{L} .\end{cases}
$$

Therefore the average value of $w_{t}$ on $J$ is $2^{N \alpha-d\left(I_{t}, J\right) \alpha} c^{\alpha}$ if $J \not \subset I_{t}^{L}$, and $2^{L \alpha}$ if $J \subset I_{t}^{L}$.

iv) The measure $\mu_{t}$ associated to $w_{t}$ is a dyadic doubling measure, with dyadic doubling constant $2^{\alpha} /\left(2-2^{\alpha}\right)$.

v) The weight $w_{t}$ lies in every dyadic $A_{p}$ space. Its dyadic $A_{1}$ constant is $\left(2-2^{\alpha}\right)^{-1}$. Its dyadic $A_{p}$ constant, for $1<p<\infty$, is

$$
\frac{\left(2-2^{\alpha}\right)^{-1}}{\left(2-2^{-\alpha /(p-1)}\right)^{p-1}}\left(1+\left(2^{-1-\alpha /(p-1)}\right)^{L}\left(\frac{2-2^{-1-\alpha /(p-1)}}{\left(2-2^{\alpha}\right)^{-1 /(p-1)}-1}\right)\right)^{p-1},
$$

which is less than the dyadic $A_{p}$ constant of the untruncated weight $v_{t}$.

vi) The weight $w_{t}$ lies in all dyadic reverse Hölder-q spaces such that $1<q<1 / \alpha$. Its dyadic reverse Hölder-q constant, for $1<q<1 / \alpha$, is

$$
\frac{2-2^{\alpha}}{\left(2-2^{\alpha q}\right)^{1 / q}}\left(1+\left(2^{\alpha q-1}\right)^{L}\left(\frac{2-2^{\alpha q}}{\left(2-2^{\alpha}\right)^{q}}-1\right)\right)^{1 / q}
$$

which is less than the dyadic reverse Hölder-q constant of $v_{t}$.

vii) The dyadic doubling constant, the $A_{1}^{d}$ constant, the $A_{p}^{d}$ constant, and the $R H_{q}^{d}$ constant of $w_{t}$ converge to 1 as $\alpha$ decreases to 0 .

Next we establish the above properties, first for the untruncated weight $v_{t}(x)=\left(M_{d}\left(c \delta_{t}(x)\right)\right)^{\alpha}$, and then for the weight $w_{t}(x)$ which has been truncated at level $L$.

Proof of Lemma 3.1. i) We verify that the total mass of $\nu_{t}$ is 1

$$
\begin{aligned}
\nu_{t}([0,1]) & =\int_{0}^{1} v_{t}(x) d x \\
& =\sum_{k=0}^{\infty} \int_{\mathcal{F}_{k}} v_{t}(x) d x
\end{aligned}
$$




$$
\begin{aligned}
& =\sum_{k=0}^{\infty} 2^{-k-1} 2^{k \alpha} c^{\alpha} \\
& =\frac{c^{\alpha}}{2} \sum_{k=0}^{\infty}\left(2^{\alpha-1}\right)^{k} \\
& =\frac{2-2^{\alpha}}{2} \frac{1}{1-2^{\alpha-1}} \\
& =1 .
\end{aligned}
$$

ii) If $I_{t} \in \mathcal{D}_{N}$ is the unique dyadic interval of length $2^{-N}$ which contains $t$, then $I_{t}=\cup_{k=N}^{\infty} \mathcal{F}_{k}$, and so the total mass of $I_{t}$ is

$$
\begin{aligned}
\nu_{t}\left(I_{t}\right) & =\int_{I_{t}} v_{t}(x) d x \\
& =\sum_{k=N}^{\infty} \int_{\mathcal{F}_{k}} v_{t}(x) d x \\
& =\frac{2-2^{\alpha}}{2} \frac{\left(2^{\alpha-1}\right)^{N}}{1-2^{\alpha-1}} \\
& =2^{N \alpha-N} .
\end{aligned}
$$

iii) Now suppose $J \in \mathcal{D}_{N}$ does not contain $t$. The smallest dyadic interval containing both $I_{t}$ and $J$ is $d\left(I_{t}, J\right)$ generations back. Here $1 \leq$ $d\left(I_{t}, J\right) \leq N$. Then $J \subset \mathcal{F}_{N-d\left(I_{t}, J\right)}$. By $(20), v_{t} \equiv 2^{\left(N-d\left(I_{t}, J\right)\right) \alpha} c^{\alpha}$ on $J$, and so

$$
\nu_{t}(J)=\int_{J} v_{t}=2^{-N} 2^{\left(N-d\left(I_{t}, J\right)\right) \alpha} c^{\alpha}=2^{N \alpha-d\left(I_{t}, J\right) \alpha-N} c^{\alpha} .
$$

Thus the interval $I_{t}$ containing $t$ has the largest mass in $\mathcal{D}_{N}$, and the mass of $J \in \mathcal{D}_{N}$ decreases as the generational distance of $J$ from $I_{t}$ increases.

iv) The weight $v_{t}$ is doubling on dyadic sister intervals, with maximal ratio $2^{\alpha} /\left(2-2^{\alpha}\right)$. For if $I, J \in \mathcal{D}_{N}$ have the same parent, and if neither $I$ nor $J$ contains $t$, then $I$ and $J$ are at the same generational distance from the $I_{t} \in \mathcal{D}_{N}$ which does contain $t$, and so by (24)

$$
\nu_{t}(I)=2^{N \alpha-d\left(I_{t}, I\right) \alpha-N} c^{\alpha}=2^{N \alpha-d\left(I_{t}, J\right) \alpha-N} c^{\alpha}=\nu_{t}(J) .
$$

On the other hand, if $I_{t} \ni t$, and $I_{t}$ and $J$ have the same parent, then $d\left(I_{t}, J\right)=1$ and so the worst possible ratio is

$$
\frac{\nu_{t}\left(I_{t}\right)}{\nu_{t}(J)}=\frac{2^{N \alpha-N}}{2^{N \alpha-\alpha-N} c^{\alpha}}=\frac{2^{\alpha}}{c^{\alpha}}=\frac{2^{\alpha}}{2-2^{\alpha}},
$$


as required. Further, $2^{\alpha} /\left(2-2^{\alpha}\right)$ decreases to 1 as $\alpha$ decreases to 0 .

v) Next we show that the $A_{1}^{d}$ constant of $v_{t}$ is $\left(2-2^{\alpha}\right)^{-1}$. If $J \in \mathcal{D}_{N}$ does not contain $t$, then $v_{t}$ is constant on $J$ and so

$$
\frac{1}{|J|} \int_{J} v_{t}=\operatorname{essinf}_{J} v_{t}
$$

For the interval $I_{t} \in \mathcal{D}_{N}$ which contains $t$, the mean value is

$$
\frac{1}{\left|I_{t}\right|} \int_{I_{t}} v_{t}=2^{N} 2^{N \alpha-N}=2^{N \alpha}
$$

The interval $I_{t}$ can be written as $\cup_{k=N}^{\infty} \mathcal{F}_{k}$, and on each $\mathcal{F}_{k}$ with $k \geq N$, $v_{t} \equiv 2^{k \alpha} c^{\alpha} \geq 2^{N \alpha} c^{\alpha}$, by (20). So the essential infimum of $v_{t}$ on $I_{t}$ is $2^{N \alpha} c^{\alpha}$, and we have

$$
\frac{\frac{1}{\left|I_{t}\right|} \int_{I_{t}} v_{t}}{\operatorname{ess} \inf _{I_{t}} v_{t}}=\frac{2^{N \alpha}}{2^{N \alpha} c^{\alpha}}=\frac{1}{2-2^{\alpha}}
$$

Hence $v_{t}$ is in $A_{1}^{d}$, with $A_{1}^{d}$ constant equal to $\left(2-2^{\alpha}\right)^{-1}$, as claimed. The $A_{1}^{d}$ constant $\left(2-2^{\alpha}\right)^{-1}$ decreases to 1 as $\alpha$ decreases to 0 .

Since the $A_{p}^{d}$ spaces are nested and increasing as $p \longrightarrow \infty$, the weight $v_{t}$ is actually in every $A_{p}^{d}$. We compute the $A_{p}^{d}$ constant of $v_{t}$.

First, if $J \in \mathcal{D}_{N}$, and $J$ does not contain $t$, then $v_{t}$ is constant on $J$ and so the product in the $A_{p}^{d}$ condition (15) is 1. Second, if $I_{t} \in \mathcal{D}_{N}$ contains $t$, then $I_{t}=\cup_{k=N}^{\infty} \mathcal{F}_{k}$, and on each $\mathcal{F}_{k}, v_{t} \equiv 2^{k \alpha} c^{\alpha}$. Now by $(23)$ the mean value of $v_{t}$ on $I_{t}$ is $2^{N \alpha}$. So the product in (15) is

$$
\begin{aligned}
\left(\frac{1}{\left|I_{t}\right|} \int_{I_{t}} v_{t}\right) & \left(\frac{1}{\left|I_{t}\right|} \int_{I_{t}}\left(\frac{1}{v_{t}}\right)^{1 /(p-1)}\right)^{p-1} \\
= & 2^{N \alpha}\left(2^{N} \sum_{k=N}^{\infty} \int_{\mathcal{F}_{k}}\left(\frac{1}{v_{t}}\right)^{1 /(p-1)}\right)^{p-1} \\
= & 2^{N \alpha}\left(2^{N} \sum_{k=N}^{\infty} 2^{-k-1}\left(2^{-\alpha k} c^{-\alpha}\right)^{1 /(p-1)}\right)^{p-1} \\
= & 2^{N \alpha} c^{-\alpha}\left(2^{N-1} \sum_{k=N}^{\infty}\left(2^{-1-\alpha /(p-1)}\right)^{k}\right)^{p-1} \\
= & 2^{N \alpha} c^{-\alpha}\left(2^{N} \frac{\left(2^{-1-\alpha /(p-1)}\right)^{N}}{2-2^{-\alpha /(p-1)}}\right)^{p-1} \\
= & \frac{1}{2-2^{\alpha}} \frac{1}{\left(2-2^{-\alpha /(p-1)}\right)^{p-1}} .
\end{aligned}
$$


The last expression is greater than 1 , and so it is the $A_{p}^{d}$ constant of the weight $v_{t}$. Further, the $A_{p}^{d}$ constant decreases to 1 as $\alpha$ decreases to 0 , since both its factors decrease to 1 .

vi) We now show that our weights $v_{t}$ also lie in the dyadic reverse Hölder- $q$ space $R H_{q}^{d}$, as long as $q<1 / \alpha$, and we compute their dyadic reverse Hölder- $q$ constants. For dyadic intervals $J$ which do not contain $t$, the weight $v_{t}$ is constant on $J$ and so the reverse Hölder condition (17) holds with constant 1 . For the dyadic interval $I_{t}$ of length $2^{-N}$ which contains $t$, we begin by computing the average value of $v_{t}^{q}$ on $I_{t}=\cup_{k=N}^{\infty} \mathcal{F}_{k}$

$$
\begin{aligned}
\frac{1}{\left|I_{t}\right|} \int_{I_{t}} v_{t}^{q} & =2^{N} \sum_{k=N}^{\infty} \int_{\mathcal{F}_{k}} v_{t}^{q} \\
& =2^{N} \sum_{k=N}^{\infty} 2^{k \alpha q} c^{\alpha q} 2^{-k-1} \\
& =2^{N} \frac{c^{\alpha q}}{2} \sum_{k=N}^{\infty}\left(2^{\alpha q-1}\right)^{k} \\
& =2^{N} \frac{c^{\alpha q}}{2} \frac{\left(2^{\alpha q-1}\right)^{N}}{1-2^{\alpha q-1}} \\
& =\frac{\left(2-2^{\alpha}\right)^{q}}{2-2^{\alpha q}} 2^{\alpha q N}
\end{aligned}
$$

assuming that $2^{\alpha q-1}<1$, in other words that $q<1 / \alpha$. Therefore, since the mean value of $v_{t}$ on $I_{t}$ is $2^{\alpha N}$,

$$
\left(\frac{1}{\left|I_{t}\right|} \int_{I_{t}} v_{t}^{q}\right)^{1 / q}=\frac{2-2^{\alpha}}{\left(2-2^{\alpha q}\right)^{1 / q}} \frac{1}{\left|I_{t}\right|} \int_{I_{t}} v_{t} .
$$

Since the expression $\left(2-2^{\alpha}\right) /\left(2-2^{\alpha q}\right)^{1 / q}$ is greater than 1 , it is the dyadic reverse Hölder- $q$ constant of $v_{t}$.

vii) We have already observed that the dyadic doubling constant, the $A_{1}^{d}$ constant, and the $A_{p}^{d}$ constant of $v_{t}$ decrease to 1 as $\alpha$ decreases to 0 . The numerator and denominator of the $R H_{q}^{d}$ constant $\left(2-2^{\alpha}\right) /\left(2-2^{q \alpha}\right)^{1 / q}$ of $v_{t}$ both increase to 1 as $\alpha$ decreases to 0 . The function $f(\alpha)=(2-$ $\left.2^{\alpha}\right)^{q}-\left(2-2^{q \alpha}\right)$ has $f(0)=0$ and has positive derivative for $0<\alpha<1 / q$. It follows that the $R H_{q}^{d}$ constant of $v_{t}$ decreases to 1 as $\alpha$ decreases to 0 .

In particular, we have shown that $v_{t} \in R H_{q}^{d}$ for all $q<1 / \alpha$, and that by decreasing $\alpha$ towards 0 we can both make the dyadic reverse Hölder- $q$ 
constant arbitrarily small, and make our weights lie in $R H_{q}^{d}$ spaces for arbitrarily large $q$.

This completes the proof of Lemma 3.1.

We establish the corresponding properties for the truncated weight $w_{t}$.

Proof of Lemma 3.3. i) The truncated weight $w_{t}$ has the same total mass 1 on $[0,1]$ as $v_{t}$ does, since the only change is to replace $v_{t}$ on the subinterval $I_{t}^{L}$ by its average value on $I_{t}^{L}$.

ii) The mass of $I_{t} \in \mathcal{D}_{N}$ is unchanged by truncation of the weight at level $L$, if $I_{t} \supseteq I_{t}^{L}$. However if $I_{t} \subset I_{t}^{L}$, then $w_{t} \equiv 2^{L \alpha}$ on $I_{t}$, so the mass of $I_{t}$ is $2^{L \alpha-N}$.

iii) If $t \notin J \in \mathcal{D}_{N}$ and $J \not \subset I_{t}^{L}$, then the mass of $J$ is unchanged by truncation of $v_{t}$ at level $L$. If $J \subset I_{t}^{L}$, then the mass of $J$ becomes $2^{L \alpha-N}$.

iv) The ratio $\mu_{t}\left(I_{t}\right) / \mu(J)=2^{\alpha} /\left(2-2^{\alpha}\right)$ is achieved whenever $I_{t}$ and $J$ are dyadic sisters of length $2^{-N}, I_{t} \ni t$, and $0 \leq N<L$. For all other pairs of dyadic sisters, the ratio of the masses is 1 . Therefore the dyadic doubling constant of $w_{t}$ is still $2^{\alpha} /\left(2-2^{\alpha}\right)$, as for $v_{t}$.

v) We need only consider the dyadic intervals $I_{t} \ni t$ of length $\left|I_{t}\right|=$ $2^{-N}$, where $0 \leq N<L$, since the truncated weight $w_{t}$ is constant on all other dyadic intervals.

The product in the $A_{1}^{d}$ condition (16) achieves the value $\left(2-2^{\alpha}\right)^{-1}$ for each such interval $I_{t}$, so the $A_{1}^{d}$ constant of $w_{t}$ is still $\left(2-2^{\alpha}\right)^{-1}$. The first factor in the product in the $A_{p}^{d}$ condition (15) is the mean value of $w_{t}$ on $I_{t}$, which is still $2^{N \alpha}$. Integrating $w_{t}^{-1 /(p-1)}$ on $I_{t}$,

$$
\begin{aligned}
& \int_{I_{t}}\left(\frac{1}{w_{t}}\right)^{1 /(p-1)} \\
& =\sum_{k=N}^{L-1} \int_{\mathcal{F}_{k}}\left(\frac{1}{2^{k \alpha} c^{\alpha}}\right)^{1 /(p-1)} d x+\int_{I_{t}^{L}}\left(\frac{1}{2^{L \alpha}}\right)^{1 /(p-1)} d x \\
& =\left(\frac{c^{-\alpha /(p-1)}}{2} \sum_{k=N}^{L-1}\left(2^{-1-\alpha /(p-1)}\right)^{k}\right)+\left(2^{-1-\alpha /(p-1)}\right)^{L} \\
& =\frac{c^{-\alpha /(p-1)}}{2} \frac{\left(2^{-1-\alpha /(p-1)}\right)^{N}-\left(2^{-1-\alpha /(p-1)}\right)^{L}}{1-2^{-1-\alpha /(p-1)}}+\left(2^{-1-\alpha /(p-1)}\right)^{L}
\end{aligned}
$$

$$
=\frac{\left(2-2^{\alpha}\right)^{-1 /(p-1)}}{2-2^{-\alpha /(p-1)}}\left(\left(2^{-1-\alpha /(p-1)}\right)^{N}-\left(2^{-1-\alpha /(p-1)}\right)^{L}\right)
$$




$$
\begin{aligned}
& +\left(2^{-1-\alpha /(p-1)}\right)^{L} \\
= & \frac{\left(2-2^{\alpha}\right)^{-1 /(p-1)}}{2-2^{-\alpha /(p-1)}} \\
& \cdot\left(\left(2^{-1-\alpha /(p-1)}\right)^{N}+\left(2^{-1-\alpha /(p-1)}\right)^{L}\left(\frac{2-2^{-1-\alpha /(p-1)}}{\left(2-2^{\alpha}\right)^{-1 /(p-1)}}-1\right)\right) .
\end{aligned}
$$

Hence the product in the $A_{p}^{d}$ condition (15) is

$$
\begin{aligned}
\left(\frac{1}{\left|I_{t}\right|} \int_{I_{t}} w_{t}\right)\left(\frac{1}{\left|I_{t}\right|} \int_{I_{t}}\left(\frac{1}{w_{t}}\right)^{1 /(p-1)}\right)^{p-1} \\
=\frac{\left(2-2^{\alpha}\right)^{-1}}{\left(2-2^{-\alpha /(p-1)}\right)^{p-1}} 2^{N \alpha+N(p-1)} \\
\\
\quad \cdot\left(\left(2^{-1-\alpha /(p-1)}\right)^{N}+\left(2^{-1-\alpha /(p-1)}\right)^{L}\left(\frac{2-2^{-1-\alpha /(p-1)}}{\left(2-2^{\alpha}\right)^{-1 /(p-1)}}-1\right)\right) \\
=\frac{\left(2-2^{\alpha}\right)^{-1}}{\left(2-2^{-\alpha /(p-1)}\right)^{p-1}} \\
\quad \cdot\left(1+\left(2^{-1-\alpha /(p-1)}\right)^{L-N}\left(\frac{2-2^{-1-\alpha /(p-1)}}{\left(2-2^{\alpha}\right)^{-1 /(p-1)}}-1\right)\right)^{p-1} .
\end{aligned}
$$

The first factor on the right hand side is the old $A_{p}^{d}$ constant of the untruncated weight $v_{t}$.

The second factor is positive but less than 1 , because $2-2^{-1-\alpha /(p-1)}<$ $\left(2-2^{\alpha}\right)^{-1 /(p-1)}$ for $p>1$.

Further, since $2^{-1-\alpha /(p-1)}<1$, the second factor achieves its maximum when $N=0$. Therefore the dyadic $A_{p}$ constant of the truncated weight $w_{t}$ is

$$
\frac{\left(2-2^{\alpha}\right)^{-1}}{\left(2-2^{-\alpha /(p-1)}\right)^{p-1}}\left(1+\left(2^{-1-\alpha /(p-1)}\right)^{L}\left(\frac{2-2^{-1-\alpha /(p-1)}}{\left(2-2^{\alpha}\right)^{-1 /(p-1)}}-1\right)\right)^{p-1}
$$

which is less than the dyadic $A_{p}$ constant $\left(2-2^{\alpha}\right)^{-1}\left(2-2^{\alpha /(p-1)}\right)^{-(p-1)}$ of the untruncated weight $v_{t}$, as required.

vi) When we truncate the weight $v_{t}$ at level $L$, the dyadic reverse Hölder- $q$ constant also decreases. Again, we need only consider the dyadic intervals $I_{t} \ni t$ of length $\left|I_{t}\right|=2^{-N}$, where $0 \leq N<L$, since the truncated 
weight $w_{t}$ is constant on all other dyadic intervals. The average value of $w_{t}$ on $I_{t}$ is still $2^{N \alpha}$. Now we compute the average value of $w_{t}^{q}$ on $I_{t}$

$$
\begin{aligned}
\frac{1}{\left|I_{t}\right|} \int_{I_{t}} w_{t}^{q} & =2^{N}\left(\sum_{k=N}^{L-1} \int_{\mathcal{F}_{k}}\left(2^{k \alpha} c^{\alpha}\right)^{q} d x+\int_{I_{t}^{L}}\left(2^{L \alpha}\right)^{q} d x\right) \\
& =2^{N}\left(\left(\frac{c^{\alpha q}}{2} \sum_{k=N}^{L-1}\left(2^{\alpha q-1}\right)^{k}\right)+\left(2^{\alpha q-1}\right)^{L}\right) \\
& =2^{N}\left(\frac{c^{\alpha q}}{2} \frac{\left(2^{\alpha q-1}\right)^{N}-\left(2^{\alpha q-1}\right)^{L}}{1-2^{\alpha q-1}}+\left(2^{\alpha q-1}\right)^{L}\right) \\
& =2^{N}\left(\frac{\left(2-2^{\alpha}\right)^{q}}{2-2^{\alpha q}}\left(\left(2^{\alpha q-1}\right)^{N}-\left(2^{\alpha q-1}\right)^{L}\right)+\left(2^{\alpha q-1}\right)^{L}\right) \\
& =2^{N} \frac{\left(2-2^{\alpha}\right)^{q}}{2-2^{\alpha q}}\left(\left(2^{\alpha q-1}\right)^{N}+\left(2^{\alpha q-1}\right)^{L}\left(\frac{2-2^{\alpha q}}{\left(2-2^{\alpha}\right)^{q}}-1\right)\right)
\end{aligned}
$$

Hence

$$
\begin{aligned}
\frac{\left(\frac{1}{\left|I_{t}\right|} \int_{I_{t}} w_{t}^{q}\right)^{1 / q}}{\frac{1}{\left|I_{t}\right|} \int_{I_{t}} w_{t}}= & \frac{2-2^{\alpha}}{\left(2-2^{\alpha q}\right)^{1 / q}} 2^{(N / q)-N \alpha} \\
& \cdot\left(\left(2^{\alpha q-1}\right)^{N}+\left(2^{\alpha q-1}\right)^{L}\left(\frac{2-2^{\alpha q}}{\left(2-2^{\alpha}\right)^{q}}-1\right)\right)^{1 / q} \\
= & \frac{2-2^{\alpha}}{\left(2-2^{\alpha q}\right)^{1 / q}}\left(1+\left(2^{\alpha q-1}\right)^{L-N}\left(\frac{2-2^{\alpha q}}{\left(2-2^{\alpha}\right)^{q}}-1\right)\right)^{1 / q} .
\end{aligned}
$$

The first factor on the right hand side is the old $R H_{q}^{d}$ constant of the untruncated weight $v_{t}$. The second factor is positive but less than 1 , because $2-2^{\alpha q}<\left(2-2^{\alpha}\right)^{q}$ for $q>1$. Further, since $2^{\alpha q-1}<1$, the second factor achieves its maximum when $N=0$. Therefore the dyadic reverse Hölder- $q$ constant of the truncated weight $w_{t}$ is

$$
\frac{2-2^{\alpha}}{\left(2-2^{\alpha q}\right)^{1 / q}}\left(1+\left(2^{\alpha q-1}\right)^{L}\left(\frac{2-2^{\alpha q}}{\left(2-2^{\alpha}\right)^{q}}-1\right)\right)^{1 / q}
$$

and this is less than $\left(2-2^{\alpha}\right)\left(2-2^{\alpha q}\right)^{-1 / q}$, as required.

vii) The dyadic doubling constant and the $A_{1}^{d}$ constant of $w_{t}$ are the same as those of $v_{t}$, so they decrease to 1 as $\alpha$ decreases to 0 . The $A_{p}^{d}$ 
constant, for $1<p<\infty$, and the $R H_{q}^{d}$ constant of $w_{t}$ are less than those of $v_{t}$, and so they also converge to 1 as $\alpha$ decreases to 0 . This completes the proof of Lemma 3.3.

A weight $w$ is in $A_{\infty}^{d}$ if and only if there is a constant $C$ such that

$$
\frac{1}{|I|} \int_{I} w \leq C \exp \left(\frac{1}{|I|} \int_{I} \log w\right)
$$

for all dyadic intervals $I$; the smallest such $C$ is the $A_{\infty}^{d}$ constant of $w$. Short computations, similar to those for $A_{p}^{d}$ above, show that the $A_{\infty}^{d}$ constant of the untruncated weight $v_{t}$ is $c^{-\alpha} 2^{-\alpha}$, which decreases to 1 as $\alpha$ decreases to 0 , and that the $A_{\infty}^{d}$ constant of the weight $w_{t}$ truncated at level $L$ is $\left(c^{-\alpha} 2^{-\alpha}\right)^{1-2^{-L}}$, which is less than that of $v_{t}$.

\section{Proof of Theorem 1.4.}

In this section, we prove the main result of our paper, Theorem 1.4. Here $C$ denotes a constant that may change from line to line.

We must prove that, with the definitions given in the introduction, the translation average $\mu(x)$ is not a doubling measure. Specifically, we show that for our sequence of pairs $A^{j}, B^{j}$ of non-dyadic intervals in $[0,1]$ of length $\left|A^{j}\right|=\left|B^{j}\right|=2^{-N_{j}}$, we have $\mu\left(A^{j}\right) / \mu\left(B^{j}\right) \longrightarrow \infty$ as $j \longrightarrow \infty$, where $\mu(\cdot)=\int w(x) d x$ as usual. Although our intervals $A^{j}, B^{j}$ are not adjacent, they are always separated by exactly twice the length of $A^{j}$ : for each $j$ our $B^{j}$ is the translation of $A^{j}$ to the right by $3\left|A^{j}\right|$. To show that this is sufficient, note that if $\mu$ is doubling, there is a constant $C$ such that for each pair of adjacent intervals of equal length, the mass of one is at most $C$ times the mass of the other. Let the intervals $E^{j}$ and $F^{j}$ be the translations of $A^{j}$ to the right by $\left|A^{j}\right|$ and $2\left|A^{j}\right|$ respectively. Then $\mu\left(A^{j}\right) \leq C \mu\left(E^{j}\right) \leq C^{2} \mu\left(F^{j}\right) \leq C^{3} \mu\left(B^{j}\right)$, contradicting the fact that $\mu\left(A^{j}\right) / \mu\left(B^{j}\right) \longrightarrow \infty$ as $j \longrightarrow \infty$.

Given a point $t \in[0,1]$, let $I_{t}^{j}$ denote the unique dyadic interval in $\mathcal{D}_{N_{j}}$ which contains $t$, dropping the $N$ in $I_{t}^{N_{j}}$ for ease of notation. Label the dyadic intervals in $\mathcal{D}_{N_{j}}$ immediately to the right of $I_{t}^{j}$ as $J_{t}^{j}, K_{t}^{j}, L_{t}^{j}$, and $M_{t}^{j}$, reading from left to right. When $t$ is very near the right end of $[0,1]$, some or all of $J_{t}^{j}, K_{t}^{j}, L_{t}^{j}$, and $M_{t}^{j}$ are wrapped around to the left end of $[0,1]$. The nested, decreasing subsets $\mathcal{G}_{j}$ of $[0,1]$ are defined by

$$
\mathcal{G}_{0}=[0,1],
$$


and

$$
\begin{aligned}
\mathcal{G}_{j} & =\left\{t \in \mathcal{G}_{j-1}: d\left(I_{t}^{j}, K_{t}^{j}\right)=N_{j} \beta\right\} \\
& =\left\{t \in[0,1]: d\left(I_{t}^{1}, K_{t}^{1}\right)=N_{1} \beta, \ldots, d\left(I_{t}^{j}, K_{t}^{j}\right)=N_{j} \beta\right\}
\end{aligned}
$$

for $j \geq 1$. We will see that each $\mathcal{G}_{j}$ is a non-empty, finite union of dyadic intervals.

For $t \in \mathcal{G}_{0} \backslash \mathcal{G}_{1}$, let $w_{t} \equiv 1$, so that $\mu_{t}$ is Lebesgue measure on $[0,1]$. For $t \in \mathcal{G}_{j} \backslash \mathcal{G}_{j+1}, j \geq 1$, let $w_{t}$ be the truncation at level $N_{j}$ of the weight $v_{t}(x)=\left(M_{d}\left(c \delta_{t}(x)\right)\right)^{\alpha}$, and let $\mu_{t}$ be the measure whose density is $w_{t}(x)$. In Lemma 3.3, we have explicitly computed the $\mu_{t}$-masses of dyadic intervals such as $I_{t}^{j}$ and $K_{t}^{j}$. The key idea is that since $A^{j}$ is centred at 0 , the interval $A^{j}+t$ almost coincides with the dyadic interval $I_{t}^{j} \ni t$, so we can bound $\mu\left(A^{j}\right)=\int_{0}^{1} \mu_{t}\left(A^{j}+t\right) d t$ below via $\mu_{t}\left(I_{t}^{j}\right)$. Similarly, $B^{j}+t$ almost coincides with $K_{t}^{j}$, so we can bound $\mu\left(B^{j}\right)$ above via $\mu_{t}\left(K_{t}^{j}\right)$. Moreover, for $t \in \mathcal{G}_{j} \backslash \mathcal{G}_{j+1}$, the generational distance $d\left(I_{t}^{j}, K_{t}^{j}\right)=N_{j} \beta$ is very large, and so the definition of $w_{t}$ ensures that $\mu_{t}\left(I_{t}^{j}\right)$ is much larger than $\mu_{t}\left(K_{t}^{j}\right)$.

Lemma 4.1. For $j=0,1,2, \ldots$, the measure of $\mathcal{G}_{j}$ is given by

$$
\left|\mathcal{G}_{j}\right|=2^{-N_{1} \beta-N_{2} \beta-\cdots-N_{j} \beta+j} .
$$

Proof. Fix $j$, and consider the subset $S_{l}^{j}$ of $[0,1]$ defined by

$$
S_{l}^{j}=\left\{t: d\left(I_{t}^{j}, K_{t}^{j}\right)=l\right\}
$$

The dyadic intervals $I_{t}^{j}$ and $K_{t}^{j}$ have length $2^{-N_{j}}$, and $K_{t}^{j}$ is the translation of $I_{t}^{j}$ to the right by $2\left|I_{t}^{j}\right|$. So $I_{t}^{j}$ and $K_{t}^{j}$ are never dyadic sisters.

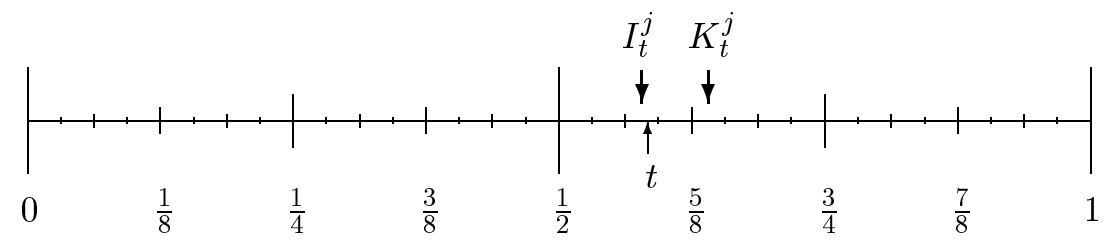

Figure 2. The collection $\mathcal{D}_{5}$ of dyadic intervals of length $2^{-5}$, showing intervals $I_{t}^{j} \ni t$ and $K_{t}^{j}$ with $d\left(I_{t}^{j}, K_{t}^{j}\right)=3$. 
The values taken by the generational distance $d\left(I_{t}^{j}, K_{t}^{j}\right)$ are the integers $l$ such that $2 \leq l \leq N_{j}$.

Let $t$ run from 0 to 1 . Half of the $2^{N_{j}}$ dyadic intervals $I_{t}^{j}$ in $\mathcal{D}_{N_{j}}$ have $d\left(I_{t}^{j}, K_{t}^{j}\right)=2$. Reading from left to right, these intervals are the first and second, fifth and sixth, ninth and tenth, and so on, up to the fourth last and third last. Half of the remaining dyadic intervals $I_{t}^{j}$ in $\mathcal{D}_{N_{j}}$ have $d\left(I_{t}^{j}, K_{t}^{j}\right)=3$; these are the third and fourth, eleventh and twelfth, and so on. Continuing in this way we see that the total length of $S_{l}^{j}$ is

$$
\left|S_{l}^{j}\right|=\left|\left\{t: d\left(I_{t}^{j}, K_{t}^{j}\right)=l\right\}\right|=2^{-l+1}, \quad \text { for } 2 \leq l \leq N_{j}-1 .
$$

The set $S_{l}^{j}$ consists of $2^{N_{j}-l+1}$ dyadic intervals of length $2^{-N_{j}}$, arranged in $2^{N_{j}-l}$ blocks of two adjacent intervals. The blocks are equally spaced around the unit circle, and each block has length $2^{-N_{j}+1}$. Therefore the distance between consecutive blocks is

$$
\frac{1-2^{-l+1}}{2^{N_{j}-l}}=2^{-N_{j}}\left(2^{l}-2\right) \text {. }
$$

When $l=N_{j}$, we have instead

$$
\left|S_{N_{j}}^{j}\right|=\left|\left\{t: d\left(I_{t}^{j}, K_{t}^{j}\right)=N_{j}\right\}\right|=2^{-l+2}=2^{-N_{j}+2}, \quad \text { for } l=N_{j} .
$$

This is because there are four intervals rather than two with $d\left(I_{t}^{j}, K_{t}^{j}\right)=$ $N_{j}$ : the two intervals $I_{t}^{j}$ immediately to the left of 1 as well as the two immediately to the left of $1 / 2$.

We prove the lemma by induction. First, $\mathcal{G}_{1}=\left\{t: d\left(I_{t}^{1}, K_{t}^{1}\right)=\right.$ $\left.N_{1} \beta\right\}=S_{N_{1} \beta}^{1}$, and the hypotheses (7) and $\beta<1$ imply that $2 \leq N_{1} \beta<N_{1}$. Hence, by equation (41),

$$
\left|\mathcal{G}_{1}\right|=2^{-N_{1} \beta+1} .
$$

Next, fix $j$ and assume that

$$
\left|\mathcal{G}_{j}\right|=2^{-N_{1} \beta-\cdots-N_{j} \beta+j} .
$$

Recall that

$$
\begin{aligned}
\mathcal{G}_{j} & =\left\{t \in[0,1]: d\left(I_{t}^{1}, K_{t}^{1}\right)=N_{1} \beta, \ldots, d\left(I_{t}^{j}, K_{t}^{j}\right)=N_{j} \beta\right\} \\
& =S_{N_{1} \beta}^{1} \cap \cdots \cap S_{N_{j} \beta}^{j},
\end{aligned}
$$


and define

$$
\widetilde{\mathcal{G}_{j+1}}=\left\{t \in[0,1]: d\left(I_{t}^{j+1}, K_{t}^{j+1}\right)=N_{j+1} \beta\right\} .
$$

So $\widetilde{\mathcal{G}_{j+1}}$ contains $\mathcal{G}_{j+1}$, and in fact $\mathcal{G}_{j+1}$ is the intersection of $\widetilde{\mathcal{G}_{j+1}}$ with $\mathcal{G}_{j}$. Since $\widetilde{\mathcal{G}_{j+1}}=S_{N_{j+1} \beta}^{j+1}$, and $2 \leq N_{j} \beta<N_{j}$, the blocks of $\widetilde{\mathcal{G}_{j+1}}$ are equally spaced around the circle, and equation (42) shows that the distance between consecutive blocks of $\widetilde{\mathcal{G}_{j+1}}$ is

$$
2^{-N_{j+1}}\left(2^{N_{j+1} \beta}-2\right) .
$$

The blocks of $\mathcal{G}_{j}$ are of length $2^{-N_{j}+1}$, and each of them contains two dyadic sister intervals of length $2^{-N_{j}}$.

The set $\mathcal{G}_{j+1}=\widetilde{\mathcal{G}_{j+1}} \cap \mathcal{G}_{j}$ will be nonempty as soon as the gaps in $\widetilde{\mathcal{G}_{j+1}}$ are shorter than the blocks of $\mathcal{G}_{j}$, and this happens as soon as $N_{j+1}$ is sufficiently large. Note that the two dyadic intervals in each block are dyadic sisters, and so the block itself is also a dyadic interval. Therefore, since dyadic intervals are either nested or disjoint, each block of $\widetilde{\mathcal{G}_{j+1}}$ that meets a block of $\mathcal{G}_{j}$ must be wholly contained in it.

Now, our hypothesis (7) implies that

$$
N_{j+1} \geq \frac{N_{1}+\cdots+N_{j}}{\alpha-\beta \alpha-\beta}>\frac{N_{j}}{1-\beta},
$$

since $\alpha-\beta \alpha<1$. Therefore

$$
2^{-N_{j+1}}\left(2^{N_{j+1} \beta}-2\right)<2^{-N_{j+1}(1-\beta)}<2^{-N_{j}}<2^{-N_{j}+1} .
$$

We have shown that the distance between consecutive blocks of $\widetilde{\mathcal{G}_{j+1}}$ is shorter than the length of the blocks in $\mathcal{G}_{j}$, and so $\mathcal{G}_{j+1}$ is nonempty.

Finally,

$$
\frac{\left|\mathcal{G}_{j+1}\right|}{2^{-N_{1} \beta-\cdots-N_{j} \beta+j}}=\frac{\left|\widetilde{\mathcal{G}_{j+1}} \cap \mathcal{G}_{j}\right|}{\left|\widetilde{\mathcal{G}_{j}}\right|}=\frac{\left|\widetilde{\mathcal{G}_{j+1}}\right|}{|[0,1]|}=2^{-N_{j+1} \beta+1},
$$

and so

$$
\left|\mathcal{G}_{j+1}\right|=2^{-N_{1} \beta-\cdots-N_{j} \beta-N_{j+1} \beta+j+1},
$$

as required. 
Lemma 4.2. The $\mu$-mass of the interval $A^{j}$ is bounded below as follows

$$
\mu\left(A^{j}\right) \geq C 2^{-N_{1} \beta-\cdots-N_{j} \beta+N_{j} \alpha-N_{j}+j},
$$

where $C$ is independent of $j$.

Proof. The key point is that $A^{j}+t$ contains at least one of the halves of $I_{t}^{j}$, and so $\mu_{t}\left(A^{j}+t\right) \geq C \mu_{t}\left(I_{t}\right)$ with a constant $C$ depending only on the dyadic doubling constant of $\mu_{t}$. Hence, using lemmas 3.3.ii) and 4.1 in the fourth line,

$$
\begin{aligned}
\mu\left(A^{j}\right) & =\int_{0}^{1} \mu_{t}\left(A^{j}+t\right) d t \\
& \geq C \int_{0}^{1} \mu_{t}\left(I_{t}^{j}\right) d t \\
& \geq C \int_{\mathcal{G}_{j} \backslash \mathcal{G}_{j+1}} \mu_{t}\left(I_{t}^{j}\right) d t \\
& \geq C 2^{-N_{1} \beta-\cdots-N_{j} \beta+j-1} 2^{N_{j} \alpha-N_{j}} \\
& =C 2^{-N_{1} \beta-\cdots-N_{j} \beta+N_{j} \alpha-N_{j}+j},
\end{aligned}
$$

as required.

Lemma 4.3. The $\mu$-mass of the interval $B^{j}$ is bounded above as follows

$$
\mu\left(B^{j}\right) \leq C 2^{-N_{1} \beta-\cdots-N_{j} \beta+N_{j} \alpha-N_{j} \beta \alpha-N_{j}+j},
$$

where $C$ is independent of $j$.

Proof. Fix $j$. We first bound $\mu\left(B^{j}\right)$ by integrals over the sets $\mathcal{G}_{k} \backslash \mathcal{G}_{k+1}$. Noting that $B^{j}+t$ lies in $K_{t}^{j} \cup L_{t}^{j} \cup M_{t}^{j}$, and that $\mu_{t}\left(K_{t}^{j}\right) \geq \mu_{t}\left(L_{t}^{j}\right) \geq \mu_{t}\left(M_{t}^{j}\right)$ because $w_{t}$ decays away from its peak at $t \in I_{t}^{j}$, we obtain

$$
\begin{aligned}
\mu\left(B^{j}\right) & =\int_{0}^{1} \mu_{t}\left(B^{j}+t\right) d t \\
& \leq \int_{0}^{1} \mu_{t}\left(K_{t}^{j}\right)+\mu_{t}\left(L_{t}^{j}\right)+\mu_{t}\left(M_{t}^{j}\right) d t \\
& \leq 3 \int_{0}^{1} \mu_{t}\left(K_{t}^{j}\right) d t
\end{aligned}
$$




$$
\begin{aligned}
& =3 \int_{\mathcal{G}_{0} \backslash \mathcal{G}_{1}} \mu_{t}\left(K_{t}^{j}\right) d t+3 \sum_{k=1}^{\infty} \int_{\mathcal{G}_{k} \backslash \mathcal{G}_{k+1}} \mu_{t}\left(K_{t}^{j}\right) d t \\
& \leq 3 \cdot 2^{-N_{j}}+3 \sum_{k=1}^{\infty} \int_{\mathcal{G}_{k} \backslash \mathcal{G}_{k+1}} \mu_{t}\left(K_{t}^{j}\right) d t .
\end{aligned}
$$

The last inequality holds because, for $t \in \mathcal{G}_{0} \backslash \mathcal{G}_{1}$, we have $w_{t} \equiv 1$ and so $\mu_{t}\left(K_{t}^{j}\right)=\left|K_{t}^{j}\right|=2^{-N_{j}}$.

Next, we bound $\mu_{t}\left(K_{t}^{j}\right)$ for $t \in \mathcal{G}_{k} \backslash \mathcal{G}_{k+1}, k>0$. We need a preliminary lemma.

Lemma 4.4. Fix $j$. For $t \in \mathcal{G}_{k}, k=1,2, \ldots$, the $\mu_{t}$-mass of the interval $K_{t}^{j}$ is bounded above as follows

$$
\mu_{t}\left(K_{t}^{j}\right) \leq \begin{cases}2^{N_{j} \alpha-N_{j} \beta \alpha-N_{j}}, & \text { if } k \geq j, \\ 2^{N_{k} \alpha-N_{j}}, & \text { if } 1 \leq k<j .\end{cases}
$$

The quantity $2^{-N_{j} \beta \alpha}$ which appears here is the key to the whole paper. It leads to the result $\mu\left(A^{j}\right) / \mu\left(B^{j}\right) \geq C 2^{N_{j} \beta \alpha}$.

Proof. The truncated weight $w_{t}$ is identically equal to $2^{N_{k} \alpha}$ on $I_{t}^{k}$. Since $t \in \mathcal{G}_{k}$, we have

$$
d\left(I_{t}^{1}, K_{t}^{1}\right)=N_{1} \beta, \ldots, d\left(I_{t}^{k}, K_{t}^{k}\right)=N_{k} \beta .
$$

If $k \geq j$, then $d\left(I_{t}^{j}, K_{t}^{j}\right)=N_{j} \beta$, and $K_{t}^{j}$ is disjoint from $I_{t}^{k}$ since $I_{t}^{k} \subset I_{t}^{j}$.

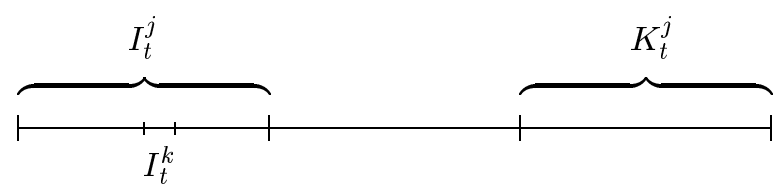

Figure 3. Here $k \geq j$, and the weight $w_{t}$ is constant on the small interval $I_{t}^{k}$ inside $I_{t}^{j}$.

We estimate the $\mu_{t}$-mass of the interval $K_{t}^{j}$ by applying the first case of Lemma 3.3.iii), with $J=K_{t}^{j}, I_{t}=I_{t}^{j}, I_{t}^{L}=I_{t}^{k}, L=N_{k}$, and $N=N_{j}$. We obtain

$$
\begin{aligned}
\mu_{t}\left(K_{t}^{j}\right) & =2^{N_{j} \alpha-d\left(I_{t}^{j}, K_{t}^{j}\right) \alpha-N_{j}} c^{\alpha} \\
& =2^{N_{j} \alpha-N_{j} \beta \alpha-N_{j}} c^{\alpha} \\
& \leq 2^{N_{j} \alpha-N_{j} \beta \alpha-N_{j}},
\end{aligned}
$$

since $c^{\alpha}=2-2^{\alpha}<1$. 
Now suppose $1 \leq k<j$, so $I_{t}^{k}$ contains $I_{t}^{j}$. There are two cases. If $I_{t}^{k}$ also contains $K_{t}^{j}$, then applying the second case of Lemma 3.3.iii) we obtain $\mu_{t}\left(K_{t}^{j}\right)=2^{N_{k} \alpha-N_{j}}$, as required.

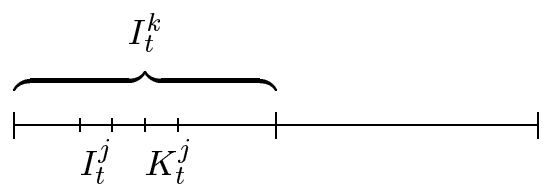

Figure 4. Here $1 \leq k<j$, the weight $w_{t}$ is constant on the large interval $I_{t}^{k}$, and $K_{t}^{j}$ lies in $I_{t}^{k}$.

If, however, $t$ is so close to the right endpoint of $I_{t}^{k}$ that $K_{t}^{j}$ lies to the right of $I_{t}^{k}$, then the constant value of $w_{t}$ on $K_{t}^{j}$ is less than the value $2^{N_{k} \alpha}$ of $w_{t}$ on $I_{t}^{k}$, by the definition of $w_{t}$.

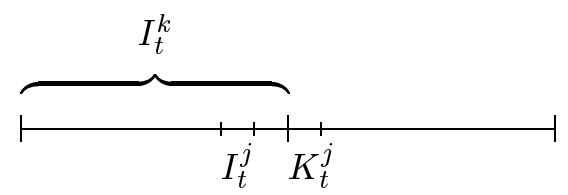

Figure 5. Here $1 \leq k<j$, the weight $w_{t}$ is constant on the large interval $I_{t}^{k}$, and $K_{t}^{j}$ lies outside $I_{t}^{k}$.

Therefore

$$
\mu_{t}\left(K_{t}^{j}\right)=\int_{K_{t}^{j}} w_{t}(x) d x \leq 2^{N_{k} \alpha-N_{j}}
$$

as required.

Proof of Lemma 4.3, continued. We consider the cases $k=j, k>j$, and $1 \leq k<j$ separately. When $k=j$, Lemmas 4.1 and 4.4 imply that

$$
\begin{aligned}
\int_{\mathcal{G}_{j} \backslash \mathcal{G}_{j+1}} \mu_{t}\left(K_{t}^{j}\right) d t & \leq\left|\mathcal{G}_{j}\right| 2^{N_{j} \alpha-N_{j} \beta \alpha-N_{j}} \\
& =2^{-N_{1} \beta-\cdots-N_{j} \beta+N_{j} \alpha-N_{j} \beta \alpha-N_{j}+j} .
\end{aligned}
$$

Next, when $k>j$, the integral over $\mathcal{G}_{k} \backslash \mathcal{G}_{k+1}$ is bounded by $2^{-k} C_{j}$, where

$$
C_{j}=2^{-N_{1} \beta-\cdots-N_{j} \beta+N_{j} \alpha-N_{j} \beta \alpha-N_{j}+j}
$$


is the exponential factor on the right hand side of the desired inequality in Lemma 4.3. For by Lemma 4.4,

$$
\mu_{t}\left(K_{t}^{j}\right) \leq 2^{N_{j} \alpha-N_{j} \beta \alpha-N_{j}},
$$

and so by Lemma 4.1 on the size of $\mathcal{G}_{k}$,

$$
\begin{aligned}
\int_{\mathcal{G}_{k} \backslash \mathcal{G}_{k+1}} \mu_{t}\left(K_{t}^{j}\right) d t & \leq 2^{-N_{1} \beta-\cdots-N_{k} \beta+k+N_{j} \alpha-N_{j} \beta \alpha-N_{j}} \\
& =2^{-N_{j+1} \beta-\cdots-N_{k} \beta+k-j} C_{j} .
\end{aligned}
$$

We claim that the first factor in the last expression is at most $2^{-k}$. To see this, first note that our hypotheses $N_{1} \geq 1 / \beta, N_{2} \geq 2 / \beta$, and $N_{j} \geq$ $(\alpha-\beta \alpha-\beta)^{-1}\left(N_{1}+\cdots+N_{j-1}\right)$ imply by induction that

$$
N_{j} \geq \frac{j+1}{\beta}, \quad \text { for } j=1,2,3, \ldots
$$

To prove our claim, it is enough to show that

$$
2 k-j \leq N_{j+1} \beta+\cdots+N_{k} \beta .
$$

The right hand side is

$$
\begin{aligned}
N_{j+1} \beta+\cdots+N_{k} \beta & \geq(j+2)+\cdots+(k+1) \\
& =\frac{(k+1)(k+2)}{2}-\frac{(j+1)(j+2)}{2} \\
& =\frac{k^{2}+3 k-j^{2}-3 j}{2} \\
& =\frac{k(k-1)-(j+1) j}{2}+2 k-j \\
& \geq 2 k-j,
\end{aligned}
$$

as required, since $k \geq j+1$.

Now suppose $1 \leq k<j$. Again, we show that the integral over $\mathcal{G}_{k} \backslash \mathcal{G}_{k+1}$ is at most $2^{-k} C_{j}$. By Lemma 4.4 , we know $\mu_{t}\left(K_{t}^{j}\right) \leq 2^{N_{k} \alpha-N_{j}}$. Therefore, by Lemma 4.1,

$$
\begin{aligned}
\int_{\mathcal{G}_{k} \backslash \mathcal{G}_{k+1}} \mu_{t}\left(K_{t}^{j}\right) d t \leq & 2^{-N_{1} \beta-\cdots-N_{k} \beta+k+N_{k} \alpha-N_{j}} \\
= & 2^{N_{k+1} \beta+\cdots+N_{j} \beta+N_{k} \alpha-N_{j} \alpha+N_{j} \beta \alpha+k-j} \\
& \cdot 2^{-N_{1} \beta-\cdots-N_{j} \beta+N_{j} \alpha-N_{j} \beta \alpha-N_{j}+j} .
\end{aligned}
$$


The second factor on the right hand side is $C_{j}$. We claim that the first factor is at most $2^{-k}$. It suffices to show that

$$
\left(N_{k+1}+\cdots+N_{j-1}\right) \beta+N_{k} \alpha+2 k-j \leq N_{j}(\alpha-\beta \alpha-\beta) .
$$

Note that $k-j<0$, and that $k \leq N_{k+1} \beta<N_{k+1} \alpha$. Also, since $\beta+\alpha<1$, our hypothesis (7) implies that

$$
N_{j} \geq \frac{\beta+\alpha}{\alpha-\beta \alpha-\beta}\left(N_{1}+\cdots+N_{j-1}\right) .
$$

Therefore

$$
\begin{aligned}
\left(N_{k+1}+\cdots+N_{j-1}\right) \beta & +N_{k} \alpha+2 k-j \\
\leq & \left(N_{k+1}+\cdots+N_{j-1}\right) \beta+\left(N_{k}+N_{k+1}\right) \alpha \\
\leq & \left(N_{1}+\cdots+N_{j-1}\right)(\beta+\alpha) \\
\leq & N_{j}(\alpha-\beta \alpha-\beta),
\end{aligned}
$$

establishing (69).

We have shown that when $k=j$, the integral of $\mu_{t}\left(K_{t}^{j}\right)$ over $\mathcal{G}_{k} \backslash \mathcal{G}_{k+1}$ is at most $C_{j}$, and when $k \neq j$, the integral is at most $2^{-k} C_{j}$. From (56), we conclude that

$$
\begin{aligned}
\mu\left(B^{j}\right) & \leq 3 \cdot 2^{-N_{j}}+3 \sum_{k=1}^{\infty} \int_{\mathcal{G}_{k} \backslash \mathcal{G}_{k+1}} \mu_{t}\left(K_{t}^{j}\right) d t \\
& \leq 3 \cdot 2^{-N_{j}}+6 C_{j} \\
& =3 \cdot 2^{-N_{j}}+6 \cdot 2^{-N_{1} \beta-\cdots-N_{j-1} \beta+N_{j}(\alpha-\beta \alpha-\beta)+j} \\
& \leq 9 \cdot 2^{-N_{1} \beta-\cdots-N_{j-1} \beta+N_{j}(\alpha-\beta \alpha-\beta)+j} .
\end{aligned}
$$

The last inequality holds because (7) implies that

$$
N_{j} \geq N_{1} \beta+\cdots+N_{j-1} \beta \text {. }
$$

This proves Lemma 4.3, with constant $C=9$.

Incidentally, truncation of the weight was crucial in controlling the case $1 \leq k<j$. For the original weight $v_{t}(x)=\left(M_{d}\left(c \delta_{t}(x)\right)\right)^{\alpha}$, the second inequality $\mu_{t}\left(K_{t}^{j}\right) \leq 2^{N_{k} \alpha-N_{j}}$ in Lemma 4.4 does not hold, and indeed in Lemma 4.3 the integral of $\mu_{t}\left(K_{t}^{j}\right)$ over $\mathcal{G}_{k} \backslash \mathcal{G}_{k+1}$ is no longer bounded by 
$2^{-k} C_{j}$. The effect of truncation is to increase $\mu_{t}\left(I_{t}^{j}\right)$ somewhat and, in most cases, to decrease $\mu_{t}\left(K_{t}^{j}\right)$ significantly.

Finally, by Lemmas 4.2 and 4.3 ,

$$
\frac{\mu\left(A^{j}\right)}{\mu\left(B^{j}\right)} \geq C \frac{2^{-N_{1} \beta-\cdots-N_{j} \beta+N_{j} \alpha-N_{j}+j}}{2^{-N_{1} \beta-\cdots-N_{j} \beta+N_{j} \alpha-N_{j} \beta \alpha-N_{j}+j}}=C 2^{N_{j} \beta \alpha},
$$

which tends to infinity as $j \longrightarrow \infty$. Therefore the translation average $\mu$ is not a doubling measure. This proves Theorem 1.4, and hence theorems $1.1,1.2$, and 1.3 .

\section{References.}

[B] Buckley, S. M., Summation conditions on weights. Michigan Math. J. 40 (1993), 153-170.

[CF] Colfman, R. R., Fefferman, C., Weighted norm inequalities for maximal functions and singular integrals. Studia Math. 51 (1974), 241-250.

[CR] Coifman, R. R., Rochberg, R., Another characterization of BMO. Proc. Amer. Math. Soc. 79 (1980), 249-254.

[FKP] Fefferman, R. A., Kenig, C. E., Pipher, J., The theory of weights and the Dirichlet problem for elliptic equations. Ann. of Math. 134 (1991), 65-124.

[GC-RF] Garcia-Cuerva, J., Rubio de Francia, J. L., Weighted norm inequalities and related topics. North Holland, 1985.

[GJ] Garnett, J. B., Jones, P. W., BMO from dyadic BMO. Pacific J. Math. 99 (1982), 351-371.

[HMW] Hunt, R. A., Muckenhoupt, B., Wheeden, R. L., Weighted norm inequalities for the conjugate function and Hilbert transform. Trans. Amer. Math. Soc. 176 (1973), 227-251.

[M] Muckenhoupt, B., Weighted norm inequalities for the Hardy maximal function. Trans. Amer. Math. Soc. 165 (1972), 207-226.

[NTV] Nazarov, F., Treil, S., Volberg, A., Cauchy integral and CalderónZygmund operators on nonhomogeneous spaces. Internat. Math. Res. Notices 15 (1997), 703-726. 
[P] Petermichl, S., Dyadic shifts and a logarithmic estimate for Hankel operators with matrix symbol. C. R. Acad. Sci. Paris Sér. I Math. 330 (2000), 455-460.

[PP] Petermichl, S., Pott, S., A version of Burkholder's theorem for operator-weighted spaces. Preprint, 2000.

Recibido: 20 de septiembre de 2000

Lesley A. Ward*

Department of Mathematics

Harvey Mudd College Claremont, CA 91711, U.S.A. ward@math.hmc.edu

\footnotetext{
* Research supported in part by the Research Fund of Harvey Mudd College.
} 